\title{
Characteristics and Clinical Outcome of Breast Cancer Patients with Asymptomatic Brain Metastases
}

\author{
Elena Laakmann ${ }^{1}$, Isabell Witzel ${ }^{1}$, Tanja Neunhöffer ${ }^{2}$, Rudolf Weide ${ }^{3}$, Marcus Schmidt ${ }^{4}$, \\ Tjoung-Won Park-Simon ${ }^{5}$ (D), Volker Möbus ${ }^{6}$, Christoph Mundhenke ${ }^{7,8}$, Arkadius Polasik ${ }^{9}$, \\ Kristina Lübbe ${ }^{10}$, Tobias Hesse ${ }^{11}$, Kerstin Riecke ${ }^{1}$, Marc Thill ${ }^{12}$, Peter A. Fasching ${ }^{13}$ (D), \\ Carsten Denkert ${ }^{14}$, Tanja Fehm ${ }^{15}$, Valentina Nekljudova ${ }^{16}$, Julia Rey ${ }^{16}$, Sibylle Loibl ${ }^{16}$ \\ and Volkmar Müller $1, *$ (D)
}

1 Department of Gynecology, Martinistraße 52, University Medical Center Hamburg-Eppendorf, 20246 Hamburg, Germany; e.laakmann@uke.de (E.L.); iwitzel@uke.de (I.W.); k.riecke@uke.de (K.R.) Frauenärzte am Dom, Rheinstraße 33, 55116 Mainz, Germany; info@frauenaerzte-am-dom.de

3 Oncological Outpatient Department, Neversstraße 5, 56068 Koblenz, Germany; weide@onkologie-koblenz.de

4 Department of Gynecology, University Medical Center of the Johannes Gutenberg University Mainz, Langenbeckstr. 1, 55131 Mainz, Germany; Marcus.Schmidt@unimedizin-mainz.de

5 Hannover Medical School, Department of Gynecology, Carl-Neuberg-Straße 1, 30625 Hannover, Germany; Park-Simon.Tjoung-Won@mh-hannover.de

6 Department of Hematology and Oncology, University Hospital Frankfurt am Main, Theodor-Stern-Kai 7, 60590 Frankfurt am Main, Germany; Volker.Moebus@kgu.de

7 Department of Gynecology, Clinic Bayreuth, Preuschwitzer Str. 101, 95445 Bayreuth, Germany; christoph.mundhenke@klinikum-Bayreuth.de

8 Department of Gynecology, University Medical Center Schleswig-Holstein, Arnold-Heller-Straße 3, 24105 Kiel, Germany

9 Department of Gynecology and Obstetrics, University Medical Center Ulm, Prittwitzstr. 43, 89075 Ulm, Germany; arkadius.polasik@uniklinik-ulm.de

10 Diakovere Henriettenstift, Breast Center, Schwemannstraße 17, 30559 Hannover, Germany; Kristina.Luebbe@diakovere.de

11 Agaplesion Diakonie Clinic Rotenburg, Department of Gynecology, Elise-Averdieck-Straße 17, 27356 Rotenburg, Germany; Hesse@diako-online.de

12 Department of Gynecology and Gynecological Oncology, Agaplesion Markus Hospital, Wilhelm-Epstein-Str. 4, 60341 Frankfurt am Main, Germany; Marc.Thill@fdk.info

13 University Hospital Erlangen, Comprehensive Cancer Center Erlangen-EMN, Department of Gynecology and Obstetrics, Friedrich-Alexander University Erlangen-Nuremberg, Universitätsstraße 21-23, 91054 Erlangen, Germany; Peter.Fasching@uk-erlangen.de

14 Institute of Pathology, University Hospital Marburg, Baldingerstraße, 35043 Marburg, Germany; carsten.denkert@uni-marburg.de

15 Department of Gynecology, University Hospital Düsseldorf, Moorenstr. 5, 40225 Düsseldorf, Germany; Tanja.Fehm@med.uni-duesseldorf.de

16 German Breast Group, Martin-Behaim-Straße 12, 63263 Neu-Isenburg, Germany; Valentina.Nekljudova@gbg.de (V.N.); Julia.Rey@gbg.de (J.R.); Sibylle.Loibl@gbg.de (S.L.)

* Correspondence: v.mueller@uke.de; Tel.: +49-40-7410-52510

Received: 13 September 2020; Accepted: 26 September 2020; Published: 28 September 2020

Simple Summary: The prognosis for patients with breast cancer that has spread to the brain is poor, and survival for these women hasn't improved over the last few decades. We do not currently test for asymptomatic brain metastases in breast cancer patients, although this does happen in some other types of cancer. In this study we wanted to find out more about breast cancer that has spread to the brain and in particular to see whether there might be any advantage to spotting brain metastases before the development of neurological symptoms. Overall, our results suggest that women could be better off if their brain metastases are diagnosed before they begin to cause symptoms. We now need to carry out a clinical trial to see what happens if we screen high-risk breast cancer patients for brain 
metastases. This will verify whether doing so could increase survival, symptom control or quality of life.

\begin{abstract}
Background: Brain metastases (BM) have become a major challenge in patients with metastatic breast cancer. Methods: The aim of this analysis was to characterize patients with asymptomatic BM $(n=580)$ in the overall cohort of 2589 patients with BM from our Brain Metastases in Breast Cancer Network Germany (BMBC) registry. Results: Compared to symptomatic patients, asymptomatic patients were slightly younger at diagnosis (median age: 55.5 vs. 57.0 years, $p=0.01$ ), had a better performance status at diagnosis (Karnofsky index $80-100 \%: 68.4 \%$ vs. $57 \%, p<0.001$ ), a lower number of BM (>1 BM: $56 \%$ vs. $70 \%, p=0.027$ ), and a slightly smaller diameter of BM (median: 1.5 vs. $2.2 \mathrm{~cm}, p<0.001$ ). Asymptomatic patients were more likely to have extracranial metastases $(86.7 \%$ vs. $81.5 \%, p=0.003)$ but were less likely to have leptomeningeal metastasis ( $6.3 \%$ vs. $10.9 \%, p<0.001)$. Asymptomatic patients underwent less intensive BM therapy but had a longer median overall survival (statistically significant for a cohort of HER2-positive patients) compared to symptomatic patients (10.4 vs. 6.9 months, $p<0.001$ ). Conclusions: These analyses show a trend that asymptomatic patients have less severe metastatic brain disease and despite less intensive local BM therapy still have a better outcome (statistically significant for a cohort of HER2-positive patients) than patients who present with symptomatic BM, although a lead time bias of the earlier diagnosis cannot be ruled out. Our analysis is of clinical relevance in the context of potential trials examining the benefit of early detection and treatment of BM.
\end{abstract}

Keywords: brain metastases; asymptomatic; breast cancer

\title{
1. Introduction
}

The incidence of brain metastases (BM) in breast cancer patients has increased in the last decades and has become a major factor in life expectancy and quality of life for many breast cancer patients [1,2]. The evaluation of the National Cancer Register in Sweden showed that compared with the period 1998-2000, patients diagnosed with a primary breast cancer during 2001-2003 were at a 17\% increased risk of being admitted with brain metastases during follow-up, and patients diagnosed in 2004-2006 were at a $44 \%$ increased risk [3].

The retrospective analysis of Darlix et al. showed that $24.6 \%$ of patients with metastatic breast cancer develop BM in the course of the disease [4]. The risk for the development of BM differs between the breast cancer subtypes [5-8]. The literature review of Lin et al. reports a BM rate of $30-55 \%$ in patients with metastatic HER2-positive breast cancer and $25-46 \%$ in patients with metastatic triple-negative breast cancer [9]. A median overall survival (OS) of 7.4 months after diagnosis of BM was observed in our previous analysis of the Brain Metastases in Breast Cancer (BMBC) Registry [10]. The survival rate of patients with BM has not improved over the last decades. In fact, analysis of the BMBC Registry has shown shorter survival between the time periods 2010-2015 compared to 2000-2009 (median survival of 5.8 months versus 7.6 months, $p<0.0001$ ) [10].

The survival rates of patients with BM differ significantly depending on the subtype of breast cancer. Patients with a HER2-positive breast cancer show the highest OS rates (median OS of 11.6 months, 95\% CI: 10.0-13.4) in the evaluation of the BMBC Registry, and the lowest survival rates could be shown for patients with triple-negative breast cancer (4.6 months, 95\% CI: 3.9-5.4). A similar trend was also observed by further studies [11].

It remains unclear whether early detection of asymptomatic BM in breast cancer patients leads to improved clinical outcomes. Currently, BM screening is not part of the management of patients with breast cancer [12-14], although brain MRI screening is part of the clinical routine for the management of patients with lung cancer [15,16], which also has a high propensity to metastasize in the brain [17-20]. 
The aim of this retrospective analysis was to characterize the cohort of breast cancer patients with asymptomatic BM at diagnosis as well as to compare the OS data of patients with and without neurological symptoms of BM. Thus, we intended to assess the possible potential benefit of early detection of $\mathrm{BM}$ and set up a rationale for the design of prospective trials.

\section{Results}

\subsection{Characteristics of Patients with Asymptomatic BM vs. Patients with Neurological Symptoms of BM}

A total of 2589 patients were included in the analysis; 2009 had documented neurological symptoms, which would raise the suspicion of BM, and 580 did not have those neurological symptoms. The following symptoms were considered as neurological symptoms indicative or highly suggestive of BMs: headache; persistent nausea or vomiting; visual disturbance; seizure; and motor deficit/changes in motor function or coordination, mental health, or psychological disturbance.

In the overall cohort, the median age at diagnosis of BM was 57.0 years. In total, $46.4 \%$ of the patients $(n=1062)$ had a HER2-positive, $22.8 \%(n=522)$ a triple-negative, and $32.1 \%(n=750)$ a luminal-A-like/luminal B-like primary breast cancer (defined as estrogen receptor (ER) and/or progesterone receptor (PR) positive, HER2-negative). The majority of the patients presented with a good performance status at the diagnosis of BM (Karnofsky index $\geq 80 \%: n=655,59 \%$ ). Approximately one third of the patients $(30.9 \%)$ had one BM at diagnosis, $42.9 \%$ of the patients had multiple BMs $(\geq 4)$. Leptomeningeal metastasis was diagnosed in $9.9 \%(n=252)$ of the patients. The majority $(n=2139$, $82.7 \%$ ) had extracranial metastases at the time of BM diagnosis. Detailed information of the patients' characteristics is presented in Tables 1 and 2.

After diagnosis of BM, $49.9 \%$ of the patients were treated with chemotherapy ( $49.9 \%$ in the asymptomatic patients group and $49.9 \%$ in the symptomatic patients group), $13.9 \%$ of the patients received endocrine therapy $(13.6 \%$ in the group of asymptomatic and $14.1 \%$ in the group of symptomatic patients), and $36.1 \%$ of the patients received a HER2-targeted therapy (36.5\% in the group of asymptomatic and $36.0 \%$ in the group of symptomatic patients (detailed information about the systemic treatment regimens is shown in Table 3).

Local BM treatment consisted of surgery alone for $6.5 \%$ of all patients $(4.4 \%$ of asymptomatic and $7.1 \%$ of symptomatic patients), cranial radiotherapy alone for $68.0 \%$ of all patients $(74.7 \%$ of asymptomatic and $66.1 \%$ of symptomatic patients), or a combination of surgery and cranial radiotherapy for $25.5 \%$ of patients ( $21 \%$ of asymptomatic and $26.9 \%$ of symptomatic patients, Table 2 ).

Univariate analysis showed that patients with asymptomatic BM were slightly younger at diagnosis of BM than symptomatic patients (median age $55.5 \mathrm{vs.} 57.0$ years, $p=0.01$ ) and at diagnosis of primary breast cancer (median age 51.0 vs. 52.0, $p=0.042$ ), and showed a lower tumor grading of the primary tumor (G1 3.1\% vs. 1.4\%, $p=0.015$ ). Asymptomatic patients had a better performance status at BM diagnosis (Karnofsky index $80-100 \% 68.4 \%$ vs. $57 \%, p<0.001$ ), a lower number of BMs (one BM $34.1 \%$ vs. 30.1\%, $p=0.027, \geq 4$ BM: $37.7 \%$ vs. $44.4 \%$ ), a slightly smaller diameter of BM (median: 1.5 vs. $2.2 \mathrm{~cm}, p<0.001$ ), less frequent leptomeningeal metastasis $(6.3 \%$ vs. $10.9 \%, p<0.001)$, and went on to have less intensive BM therapy (combined surgery and radiotherapy: $21 \%$ vs. $26.9 \%, p=0.001$ ).

Furthermore, patients with asymptomatic BM had significantly more extracranial metastases at the time of BM diagnosis compared to patients with symptomatic BM $(86.7 \%$ vs. $81.5 \%, p=0.003)$. The most common location of extracranial metastases in patients with asymptomatic BM were bone $(54.0 \%)$, lung $(39.0 \%)$, and liver $(37.2 \%)$. Whereas, the location of extracranial metastases in symptomatic BM patients was bone (43.2\%), lung (36.1\%), and liver (34.6\%). The breast cancer subtype did not differ significantly between the two groups $(p=0.195)$.

Because of the retrospective and multicenter design of our subanalysis, the missing data could not be avoided. The most missing data is recorded for the variables performance status $(n=1478)$, diameter of BM at diagnosis $(n=729)$, type of radiotherapy, local BM therapy $(n=564$ and $n=424)$, and tumor grading $(n=273)$. 
In a multivariate logistic regression analysis with 705 available patients, patients with a lower performance status were significantly more symptomatic than patients with a performance status of $100 \%$ (Karnofsky index 80-90\%: OR $=5.38,95 \%$-CI: 3.09-9.36; 60-70\%: OR $=6.44,95 \%$-CI: 3.32-12.5; 10-50\%: 14.0, 95\%-CI: 3.96-49.7; $p<0.001$ ). Furthermore, patients with more than four BM were more frequently symptomatic than asymptomatic ( $\mathrm{OR}=2.01,95 \%$-CI: $1.12-3.61 ; p=0.019)$.

\subsection{Overall Survival of Patients with Asymptomatic vs. Symptomatic BMs}

The median time from diagnosis of breast cancer to diagnosis of BM was 36.4 months for patients with asymptomatic BM and 36.0 months for symptomatic patients ( $p=0.248$; Figure 1$)$.

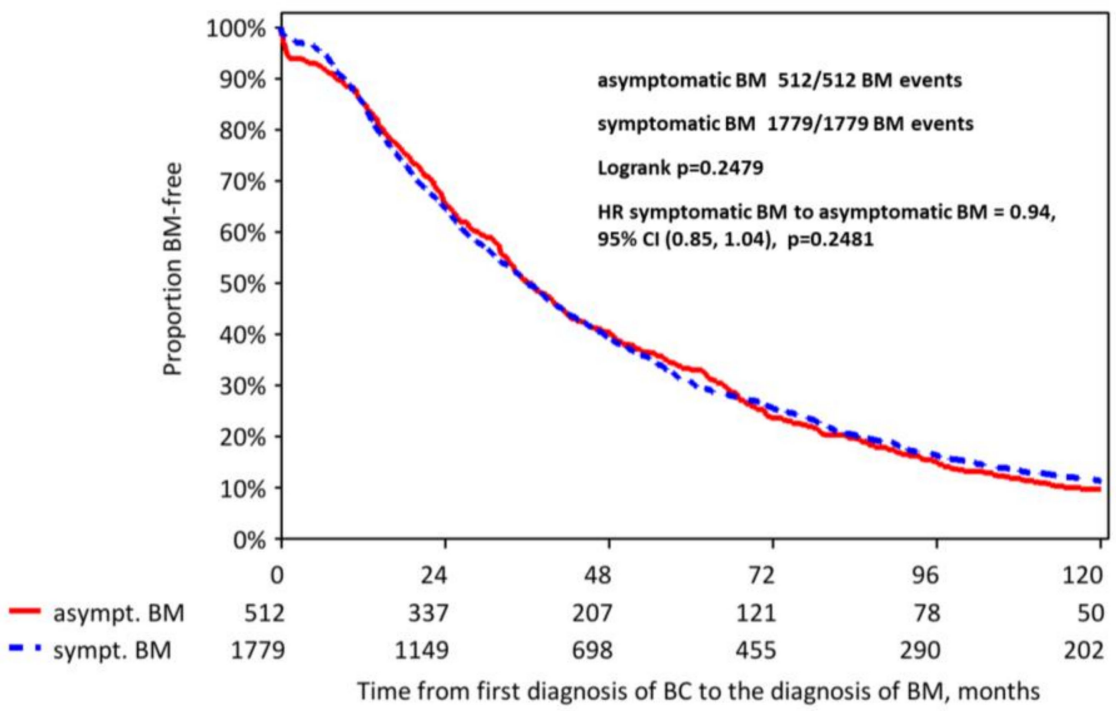

Figure 1. Kaplan-Meier curves for the time from the diagnosis of breast cancer to the diagnosis of BM.

Patients with asymptomatic BM had a longer median overall survival compared to symptomatic patients (10.4 vs. 6.9 months, $p<0.001$, Figure 2).

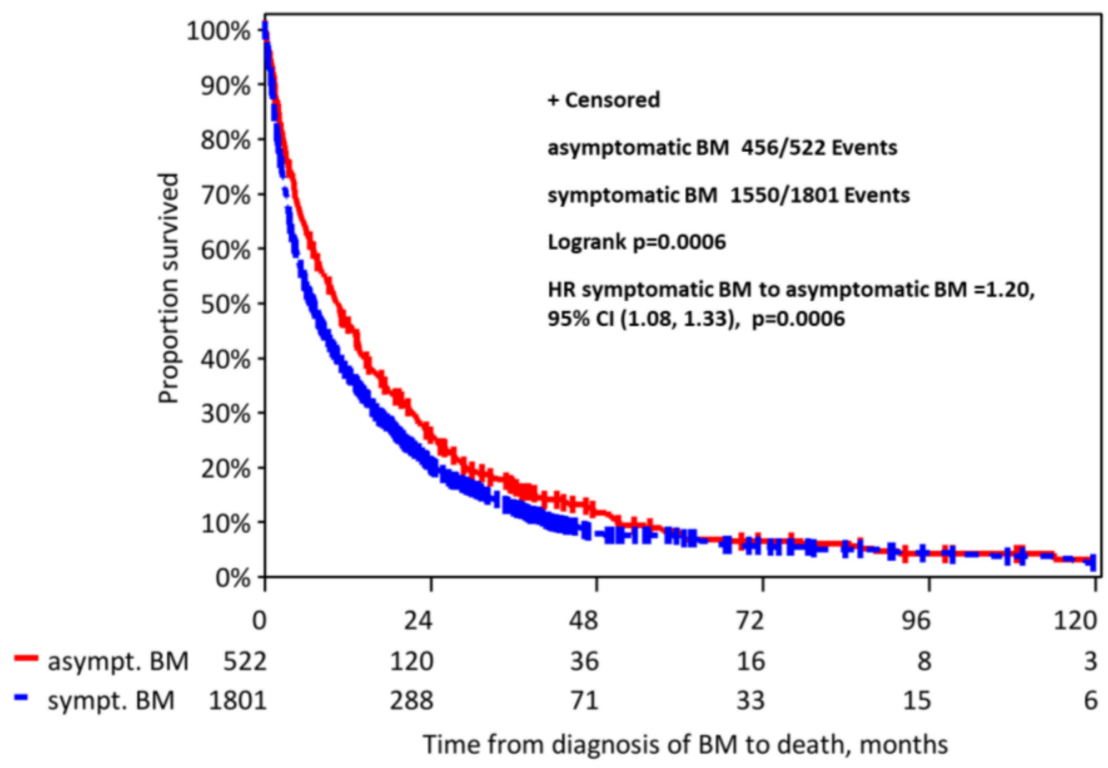

Figure 2. Kaplan-Meier curve for the time from first diagnosis of BM to death due to any reason. 
Table 1. Patients' characteristics, continuous parameters.

\begin{tabular}{|c|c|c|c|c|c|}
\hline Parameter & Category & $\begin{array}{l}\text { Asymptomatic Patients } \\
\qquad n=580\end{array}$ & $\begin{array}{c}\text { Symptomatic Patients } \\
n=2009\end{array}$ & $\begin{array}{c}\text { Overall } \\
n=2589\end{array}$ & $p$-Value \\
\hline \multirow{3}{*}{ Age at first diagnosis of $B C$, years } & Median & 51.0 & 52.0 & 52.0 & \multirow{3}{*}{0.042} \\
\hline & Min, Max & $22.0,87.0$ & $20.0,98.0$ & $20.0,98.0$ & \\
\hline & Missing & 0 & 0 & 0 & \\
\hline \multirow{3}{*}{ Age at diagnosis of BM, years } & Median & 55.5 & 57.0 & 57.0 & \multirow{3}{*}{0.010} \\
\hline & Min, Max & $26.0,87.0$ & $22.0,99.0$ & $22.0,99.0$ & \\
\hline & Missing & 0 & 0 & 0 & \\
\hline \multirow{3}{*}{ Max diameter of BM $(\mathrm{cm})$} & Median & 1.5 & 2.2 & 2.0 & \multirow{3}{*}{$<0.001$} \\
\hline & Min, Max & $0.0,22.0$ & $0.0,85.0$ & $0.0,85.0$ & \\
\hline & Missing & 200 & 529 & 729 & \\
\hline
\end{tabular}

Table 2. Patients' characteristics, categorical parameters.

\begin{tabular}{|c|c|c|c|c|c|}
\hline Parameter & Category & $\begin{array}{c}\text { Asymptomatic Patients } \\
n=580(\%)\end{array}$ & $\begin{array}{c}\text { Symptomatic Patients } \\
n=2009(\%)\end{array}$ & $\begin{array}{c}\text { Overall } \\
n=2589\end{array}$ & $p$-Value \\
\hline \multirow{4}{*}{ Histological tumor type } & ductal or ductal-lobular-invasive & $415(72.3)$ & $1461(73.5)$ & $1876(73.3)$ & \multirow{4}{*}{0.774} \\
\hline & lobular-invasive & $43(7.5)$ & $151(7.6)$ & $194(7.6)$ & \\
\hline & other & $116(20.2)$ & $375(18.9)$ & 491 (19.2) & \\
\hline & missing & 6 & 22 & 28 & \\
\hline \multirow{4}{*}{ Tumor grading } & G1 & $16(3.1)$ & $26(1.4)$ & $42(1.8)$ & \multirow{4}{*}{0.015} \\
\hline & G2 & $214(42.1)$ & $713(39.4)$ & $927(40.0)$ & \\
\hline & G3 & $278(54.7)$ & $1069(59.1)$ & $1347(58.2)$ & \\
\hline & missing & 72 & 201 & 273 & \\
\hline \multirow{4}{*}{ Biological subtype } & TNBC & $103(19.5)$ & 419 (23.2) & $522(22.4)$ & \multirow{4}{*}{0.195} \\
\hline & Luminal A-like and Luminal B-like & $178(33.7)$ & $572(31.7)$ & $750(32.1)$ & \\
\hline & Her2+ & $247(46.8)$ & $815(45.1)$ & $1062(45.5)$ & \\
\hline & missing & 52 & 203 & 255 & \\
\hline \multirow{6}{*}{ Karnofsky index at diagnosis of BM } & $100 \%$ & $60(31.6)$ & $94(10.2)$ & $154(13.9)$ & \multirow{6}{*}{$<0.001$} \\
\hline & $80-90 \%$ & $70(36.8)$ & $431(46.8)$ & $501(45.1)$ & \\
\hline & $60-70 \%$ & $46(24.2)$ & $276(30.0)$ & $322(29.0)$ & \\
\hline & $40-50 \%$ & $13(6.8)$ & $90(9.8)$ & $103(9.3)$ & \\
\hline & $10-30 \%$ & $1(0.5)$ & $30(3.3)$ & $31(2.8)$ & \\
\hline & missing & 390 & 1088 & 1478 & \\
\hline \multirow{4}{*}{ Number of BM } & 1 & $170(34.1)$ & $548(30.1)$ & $718(30.9)$ & \multirow{4}{*}{0.027} \\
\hline & $2-3$ & $141(28.3)$ & $466(25.6)$ & $607(26.1)$ & \\
\hline & $\geq 4$ & $188(37.7)$ & $809(44.4)$ & $997(42.9)$ & \\
\hline & missing & 81 & 186 & 267 & \\
\hline
\end{tabular}


Table 2. Cont

\begin{tabular}{|c|c|c|c|c|c|}
\hline Parameter & Category & $\begin{array}{c}\text { Asymptomatic Patients } \\
n=580(\%)\end{array}$ & $\begin{array}{c}\text { Symptomatic Patients } \\
n=2009(\%)\end{array}$ & $\begin{array}{c}\text { Overall } \\
n=2589\end{array}$ & $p$-Value \\
\hline \multirow{5}{*}{ Diagnostic method to detect BM } & only clinical & $19(3.5)$ & $57(2.9)$ & $76(3.0)$ & \multirow{5}{*}{$<0.001$} \\
\hline & $\mathrm{CT}^{1}$ & $108(20.1)$ & 425 (21.5) & $533(21.2)$ & \\
\hline & MRI $^{1}$ & $382(71.0)$ & $1283(64.8)$ & $1665(66.1)$ & \\
\hline & $\mathrm{CT}$ and $\mathrm{MRI}^{1}$ & $29(5.4)$ & $215(10.9)$ & $244(9.7)$ & \\
\hline & missing & 42 & 29 & 71 & \\
\hline \multirow{4}{*}{ Local treatment of BM } & Surgery only & $21(4.4)$ & $119(7.1)$ & $140(6.5)$ & \multirow{4}{*}{0.001} \\
\hline & Radiotherapy only & $360(74.7)$ & $1112(66.1)$ & $1472(68.0)$ & \\
\hline & Surgery and radiotherapy & $101(21.0)$ & $452(26.9)$ & $553(25.5)$ & \\
\hline & missing & 98 & 326 & 424 & \\
\hline \multirow{5}{*}{ Type of local radiotherapy of BM } & Whole brain radiotherapy only & $349(75.7)$ & $1265(80.9)$ & $1614(79.7)$ & \multirow{5}{*}{$<0.001$} \\
\hline & Stereotactic therapy only & $52(11.3)$ & $182(11.6)$ & 234 (11.6) & \\
\hline & Whole brain radiotherapy and stereotactic radiotherapy & $33(7.2)$ & $88(5.6)$ & $121(6.0)$ & \\
\hline & Type of radiotherapy unknown & $27(5.9)$ & $29(1.9)$ & $56(2.8)$ & \\
\hline & missing & 119 & 445 & 564 & \\
\hline \multirow{3}{*}{ Extracranial metastases at time of BM diagnosis } & yes & $502(86.7)$ & $1637(81.5)$ & $2139(82.7)$ & \multirow{3}{*}{0.003} \\
\hline & no & 77 (13.3) & $371(18.5)$ & 448 (17.3) & \\
\hline & missing & 1 & 1 & 2 & \\
\hline \multicolumn{6}{|l|}{ Localization of first metastasis if extracranial } \\
\hline \multirow{3}{*}{ bone metastases } & no & $267(46.0)$ & $1141(56.8)$ & $1408(54.4)$ & \multirow{3}{*}{$<0.001$} \\
\hline & yes & $313(54.0)$ & $868(43.2)$ & $1181(45.6)$ & \\
\hline & missing & 0 & 0 & 0 & \\
\hline \multirow{3}{*}{ liver metastases } & no & $364(62.8)$ & $1314(65.4)$ & $1678(64.8)$ & \multirow{3}{*}{0.256} \\
\hline & yes & $216(37.2)$ & $695(34.6)$ & $911(35.2)$ & \\
\hline & missing & 0 & 0 & 0 & \\
\hline \multirow{3}{*}{ lung metastases } & no & $354(61.0)$ & $1283(63.9)$ & $1637(63.2)$ & \multirow{3}{*}{0.222} \\
\hline & yes & $226(39.0)$ & $726(36.1)$ & $952(36.8)$ & \\
\hline & missing & 0 & 0 & 0 & \\
\hline \multirow{3}{*}{ skin metastases } & no & $538(92.8)$ & $1903(94.7)$ & $2441(94.3)$ & \multirow{3}{*}{0.084} \\
\hline & yes & $42(7.2)$ & $106(5.3)$ & $148(5.7)$ & \\
\hline & missing & 0 & 0 & 0 & \\
\hline \multirow{3}{*}{ other metastases } & no & $402(69.3)$ & 1468 (73.1) & $1870(72.2)$ & \multirow{3}{*}{0.082} \\
\hline & yes & 178 (30.7) & $541(26.9)$ & 719 (27.8) & \\
\hline & missing & 0 & 0 & 0 & \\
\hline \multirow{3}{*}{ Existence of leptomeningeal metastasis } & no & $523(93.7)$ & $1769(89.1)$ & $2292(90.1)$ & \multirow{3}{*}{$<0.001$} \\
\hline & yes & $35(6.3)$ & $217(10.9)$ & $252(9.9)$ & \\
\hline & missing & 22 & 23 & 45 & \\
\hline \multirow{3}{*}{ Year of BM diagnosis } & $<2010$ & $205(35.3)$ & $583(29.0)$ & 788 (30.4) & \multirow{3}{*}{0.004} \\
\hline & $\geq 2010$ & 375 (64.7) & $1426(71.0)$ & 1801 (69.6) & \\
\hline & missing & 0 & 0 & 0 & \\
\hline
\end{tabular}

${ }^{1}$ with or without clinical symptoms. 
Table 3. Breast cancer treatment after BM diagnosis (systemic treatment, endocrine therapy, targeted therapy) for patients with and without neurological symptoms.

\begin{tabular}{cccc}
\hline \multirow{2}{*}{ Treatment Specification } & Asymptomatic Patients & Symptomatic Patients & Overall \\
\cline { 2 - 4 } & $\boldsymbol{n ( \% )}$ & $\boldsymbol{n} \mathbf{( \% )}$ & $\boldsymbol{n} \mathbf{( \% )}$ \\
\hline Anthracycline & $43(4.95)$ & $107(4.99)$ & $150(4.98)$ \\
Taxane based & $66(7.59)$ & $117(5.45)$ & $183(6.07)$ \\
Taxane and Anthracycline & $108(12.43)$ & $289(13.47)$ & $397(13.17)$ \\
other chemotherapy & $217(24.97)$ & $558(26.01)$ & $775(25.71)$ \\
Tamoxifen & $18(2.07)$ & $36(1.68)$ & $54(1.79)$ \\
Aromatase inhibitor & $63(7.25)$ & $172(8.02)$ & $235(7.80)$ \\
GnRH-analoga & $9(1.04)$ & $19(0.89)$ & $28(0.93)$ \\
other hormone therapy & $28(3.22)$ & $75(3.50)$ & $103(3.42)$ \\
Trastuzumab & $85(9.78)$ & $222(10.35)$ & $307(10.19)$ \\
Trastuzumab + Pertuzumab & $9(1.04)$ & $37(1.72)$ & $46(1.53)$ \\
Lapatinib & $64(7.36)$ & $172(8.02)$ & $236(7.83)$ \\
T-DM1 & $34(3.91)$ & $79(3.68)$ & $113(3.75)$ \\
Everolimus & $6(0.69)$ & $25(1.17)$ & $31(1.03)$ \\
Bisphosphonates & $66(7.59)$ & $112(5.22)$ & $178(5.91)$ \\
Denosumab & $26(2.99)$ & $62(2.89)$ & $88(2.92)$ \\
Bevacizumab & $27(3.11)$ & $59(2.75)$ & $86(2.85)$ \\
other targeted therapy & - & $4(0.19)$ & $4(0.13)$ \\
\hline
\end{tabular}

When excluding patients with leptomeningeal metastasis from the analysis, the survival difference was less but still significant, with a median OS of asymptomatic patients of 10.4 months (95\% CI 8.97-12.8) vs. 7.8 months (95\% CI 7.10-8.67) for symptomatic patients $(p=0.012)$.

Furthermore, an analysis of OS rates in different tumor subtypes was performed (Table 4). For HER2-positive patients, a statistically significant difference between the OS of asymptomatic and symptomatic patients could be observed (Figure 3, median OS 15.2 vs. 11.5 months, $p=0.006$ ). The median OS for patients with TNBC and luminal A- and B-like patients was higher for asymptomatic patients compared to patients with neurologic complaints at BM diagnosis, though the level did not reach statistical significance ( $p=0.16$ and 0.19 , respectively).

Table 4. Median OS in different groups of biological subtypes for symptomatic and asymptomatic patients.

\begin{tabular}{ccc}
\hline Biological Subtype & Neurological Symptoms & $\begin{array}{c}\text { Median OS } \\
\mathbf{( 9 5 \%} \mathbf{C I})\end{array}$ \\
\hline TNBC & no & $6.5(4.7,9.0)$ \\
TNBC & yes & $4.1(3.4,4.8)$ \\
Luminal A and Luminal B-like & no & $8.5(5.5,10.9)$ \\
Luminal A and Luminal B-like & yes & $5.7(4.9,6.8)$ \\
HER2+ & no & $15.2(13.3,19.8)$ \\
HER2+ & yes & $11.5(10.0,13.7)$ \\
\hline
\end{tabular}




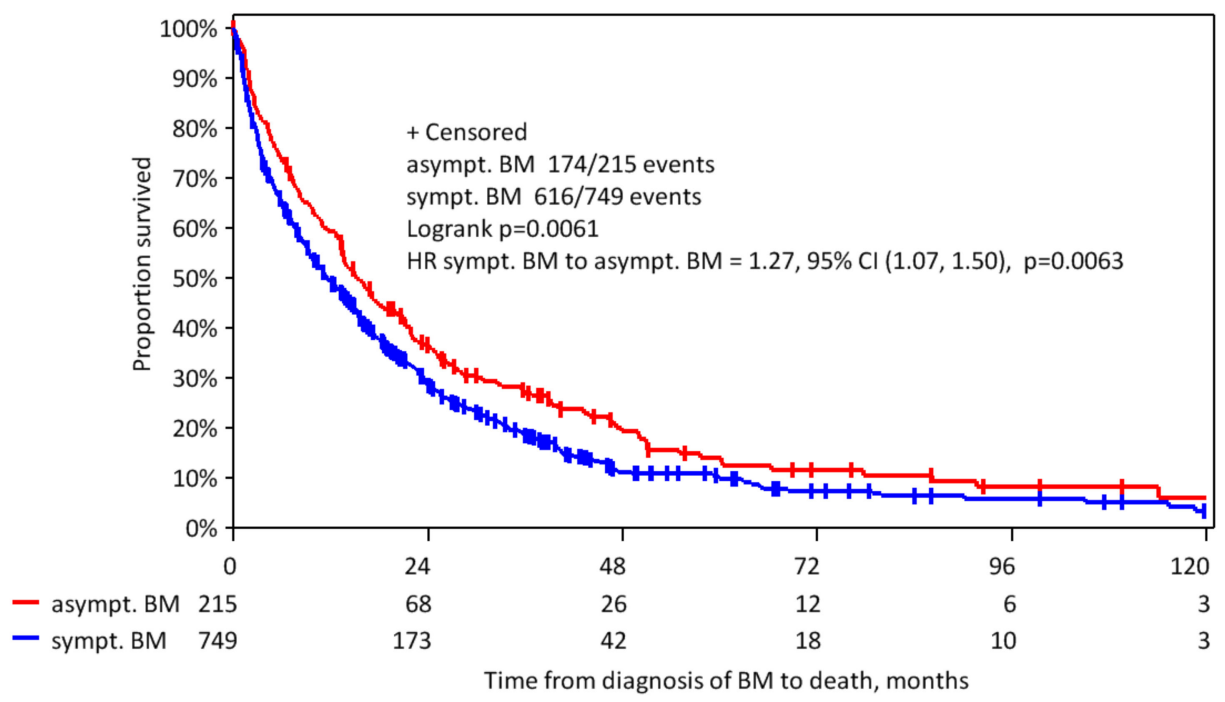

Figure 3. Kaplan-Meier curve for the time from first diagnosis of BM to death due to any reason among HER2-positive patients.

The most common cause of death in asymptomatic patients was BM and extracranial metastases $(32 \%)$, followed by BM only (25.9\%). Most of the symptomatic patients (33.1\%) died of BM, followed by brain and extracranial metastases $(29.1 \%)$. Detailed information concerning the causes of death is presented in Table 5.

Table 5. Causes of death in patients with asymptomatic and symptomatic BM.

\begin{tabular}{ccccc}
\hline Causes of Death & $\begin{array}{c}\text { Asymptomatic Patients } \\
\boldsymbol{n = 4 5 6 ( \% )}\end{array}$ & $\begin{array}{c}\text { Symptomatic Patients } \\
\boldsymbol{n = 1 5 5 0} \mathbf{( \% )}\end{array}$ & $\begin{array}{c}\text { Overall } \\
\boldsymbol{n}=\mathbf{2 0 0 6}(\mathbf{\%})\end{array}$ & $\boldsymbol{p}$-Value \\
\hline Brain metastases & $118(25.9)$ & $513(33.1)$ & $631(31.5)$ & 0.091 \\
Extracranial metastases & $72(15.8)$ & $229(14.8)$ & $301(15.0)$ & \\
Brain and extracranial metastase & $146(32.0)$ & $451(29.1)$ & $597(29.8)$ \\
Not known but tumor related & $27(5.9)$ & $82(5.3)$ & $109(5.4)$ \\
Not tumor related & $25(5.5)$ & $87(5.6)$ & $112(5.6)$ \\
Not known & $68(14.9)$ & $188(12.1)$ & $256(12.8)$ \\
Missing & 0 & 0 & 0 \\
\hline
\end{tabular}

\subsection{Neurologic Symptoms in Different Biological Subtypes}

The following neurological symptoms were included in this analysis: seizure, nausea/vomiting, motor deficit, headache, visual disturbance, and mental health or psychological disturbance (Table 6).

The most frequently documented neurological symptoms were changes in motor function or coordination, with $43.7 \%(n=228)$ of TNBC, $40.3 \%(n=302)$ of luminal A- and B-like, and $41 \%(n=435)$ of HER2-positive patients experiencing these symptoms at diagnosis. About one quarter of the patients $(26.0 \%)$ complained about headache at presentation of BM. The evaluation of the neurological complaints caused by BM showed a statistically significant difference among the different subtypes concerning the complaints headache, nausea/vomiting, and seizure. The highest rate of seizure could be detected in patients with the luminal A- and B-like subtype $(14.8 \%, n=111)$, while the lowest prevalence of seizure was detected in patients with TNBC breast cancer $(8.6 \%, n=45)$. The highest rate of headache could be detected in patients with triple-negative breast cancer $(28.3 \%, n=148)$; patients with the luminal A- and B-like subtype had the lowest rate of headache $(22.1 \%, n=166)$. Nausea/vomiting was most frequently documented by patients with triple-negative breast cancer $(25.7 \%, n=134)$; among luminal A and B-like patients, about $19.1 \%(n=143)$ of patients experienced these complaints. 
Table 6. Distribution of the neurological symptoms according to the biological subtypes.

\begin{tabular}{|c|c|c|c|c|c|c|c|c|c|}
\hline \multirow{2}{*}{ Symptom } & \multicolumn{2}{|c|}{ TNBC } & \multicolumn{2}{|c|}{$\begin{array}{c}\text { Luminal } \\
\text { A/Luminal B }\end{array}$} & \multicolumn{2}{|c|}{ HER2+ } & \multicolumn{2}{|c|}{ Overall } & \multirow[b]{2}{*}{$p$-Value } \\
\hline & $n$ & $\%$ & $n$ & $\%$ & $n$ & $\%$ & $n$ & $\%$ & \\
\hline \multicolumn{10}{|l|}{ Headache } \\
\hline no & 374 & 71.65 & 584 & 77.87 & 775 & 72.98 & 1733 & 74.25 & 0.019 \\
\hline yes & 148 & 28.35 & 166 & 22.13 & 287 & 27.02 & 601 & 25.75 & \\
\hline \multicolumn{10}{|c|}{ Visual disturbance } \\
\hline no & 446 & 85.44 & 649 & 86.53 & 912 & 85.88 & 2007 & 85.99 & 0.850 \\
\hline yes & 76 & 14.56 & 101 & 13.47 & 150 & 14.12 & 327 & 14.01 & \\
\hline \multicolumn{10}{|c|}{$\begin{array}{c}\text { Mental health or } \\
\text { psychological disturbance }\end{array}$} \\
\hline no & 442 & 84.67 & 622 & 82.93 & 906 & 85.31 & 1970 & 84.40 & 0.382 \\
\hline yes & 80 & 15.33 & 128 & 17.07 & 156 & 14.69 & 364 & 15.60 & \\
\hline \multicolumn{10}{|c|}{$\begin{array}{c}\text { Change in motor function } \\
\text { or coordination/motor } \\
\text { deficit }\end{array}$} \\
\hline no & 294 & 56.32 & 448 & 59.73 & 627 & 59.04 & 1369 & 58.65 & 0.450 \\
\hline yes & 228 & 43.68 & 302 & 40.27 & 435 & 40.96 & 965 & 41.35 & \\
\hline \multicolumn{10}{|c|}{ Nausea/vomiting } \\
\hline no & 388 & 74.33 & 607 & 80.93 & 828 & 77.97 & 1823 & 78.11 & 0.020 \\
\hline yes & 134 & 25.67 & 143 & 19.07 & 234 & 22.03 & 511 & 21.89 & \\
\hline \multicolumn{10}{|l|}{ Seizure } \\
\hline no & 477 & 91.38 & 639 & 85.20 & 926 & 87.19 & 2042 & 87.49 & 0.004 \\
\hline yes & 45 & 8.62 & 111 & 14.80 & 136 & 12.81 & 292 & 12.51 & \\
\hline \multicolumn{10}{|l|}{ none } \\
\hline no & 451 & 86.40 & 620 & 82.67 & 899 & 84.65 & 1970 & 84.40 & 0.188 \\
\hline yes & 71 & 13.60 & 130 & 17.33 & 163 & 15.35 & 364 & 15.60 & \\
\hline
\end{tabular}

${ }^{*}$ Chi-squared test: the $p$-values are to be understood descriptively; they were not corrected for multiple comparisons.

\section{Discussion}

Our analysis of 2589 patients with BM of breast cancer shows that patients with asymptomatic $\mathrm{BM}$ have lower severity of metastatic disease in the brain and have a better outcome despite a less intense local BM therapy compared to symptomatic patients. We additionally evaluated the OS in the following subtypes of breast cancer: HER2-positive, triple-negative, and luminal A- and B-like. Patients without neurological symptoms at BM diagnosis showed a better OS in all breast cancer subtypes: For HER-2 positive, the difference was statistically significant, while for triple-negative and luminal A- and B-like patients, statistical significance was not reached. The limited number of patients in this subgroup analysis must be considered by the interpretation of the results.

Our results correspond to previously published analyses from smaller cohorts. Maurer et al. [21] evaluated 30 asymptomatic HER2-positive breast cancer patients with BM. A better OS was observed for patients with asymptomatic BM in comparison to patients with symptomatic BM (hazard ratio $0.49,95 \%$ CI $0.25-0.94)$. A significantly higher rate of whole-brain radiotherapy only treatment $(63.7 \%$ vs. $36.7 \%, p=0.02)$ was used in the group of patients with symptomatic BM vs. patients without neurological complaints. The rate of SRS treatment was significantly higher in the group of asymptomatic patients ( $40 \%$ vs. $15.9 \%, p=0.02$ ). Due to a smaller cohort size with the HER2-positive subtype only, this treatment data is not completely comparable with our analysis. Nonetheless the results match the observation in our cohort that patients without neurological symptoms of BM had less intensive local BM therapy. Maurer et al. report a noticeably higher rate of asymptomatic patients with BM compared to our cohort, with $40.5 \%$ (30 of 74 patients with BM) not having neurological complaints. The reason for this high prevalence of asymptomatic patients could lie in a selection bias. According to the authors, the decision for screening for BM in case of metastatic extracranial disease was at the treating physician's discretion and did not follow any guidelines. 
Morikawa et al. [22] analyzed 100 HER2-positive breast cancer patients with BM. The investigators described that the absence of neurologic symptoms at BM diagnosis was significantly associated with fewer BM lesions, decreased use of whole-brain radiotherapy, and longer survival (multivariate hazard ratio $3.69 ; 95 \%$ CI 1.69-8.07). The rate of asymptomatic patients in this study corresponds to the results of our analysis. In total, $22.4 \%$ (580 of 2589 patients) did not have neurologic symptoms at the time of BM diagnosis in our cohort. Morikawa et al. reported about $23 \%$ of asymptomatic patients in their cohort of 100 patients with BM of HER2-positive breast cancer.

Both investigating groups support the planning of prospective trials evaluating the role of $\mathrm{BM}$ screening, especially in patients with HER2-positive metastatic breast cancer.

A retrospective analysis by Niikura et al. [11] of 1256 patients with $\mathrm{BM}$ of breast cancer including all subtypes of whom $262(20.9 \%)$ were asymptomatic neurologically revealed a longer OS in patients with asymptomatic BM vs. patients with neurologic symptoms of BM (hazard ratio $0.81,95 \%$ CI $0.69-0.94$, $p=0.006$ ). The difference between symptomatic and asymptomatic patients was also found in the multivariate analysis considering other prognostic factors, such as time to BM, number of BM, tumor subtype, and grading. No detailed comparison of patient's characteristics with and without neurological symptoms was published.

The HER2CLIMB Trial [23] examining the tyrosine kinase inhibitor tucatinib in addition to standard therapy allowed the inclusion of patients with stable BM. The encouraging results concerning the decreased intracranial progression rate, increased OS, as well as reduced risk of death under treatment with the novel compound tucatinib indicates a potential rationale for BM early detection strategies since patients might benefit more from specific therapies.

It is important to note, when considering the better OS rate in asymptomatic patients in our cohort, that many of the asymptomatic patients were diagnosed because of scans undertaken for staging purposes as part of clinical trials. This cohort of patients tends to have a better initial performance status when compared with patients with neurological symptoms of BM or who were not so clinically well and would not be included in those clinical trials. This fact may have influenced the analyzed OS times. Furthermore, a better performance status of asymptomatic patients may be related to an absence of neurological complaints. The exact percentage of patients who underwent brain imaging as a part of a clinical trial cannot be captured due to the retrospective multicenter design of this subanalysis.

Our evaluation of the neurological complaints caused by BM showed similar results in the subtypes of BC (TNBC, HER2-positive, luminal A- and luminal B-like). The statistical comparison showed significant differences among the breast cancer subtypes concerning the following neurologic complaints: seizure, nausea/vomiting, and headache. The highest rate of seizures was detected in luminal A- and B-like patients. Several risk factors for the development of seizures in patients with $\mathrm{BM}$ are described in the literature: For example, patients with a single BM, detection of tumoral hemorrhage, or supratentorial BM are at a higher risk [24]. Our previous analysis of the BMBC Registry showed that patients with positive estrogen or progesterone status have a lower number of BMs at diagnosis [25]. Thus, possibly a lower number of BMs in luminal-like patients could explain the higher probability of seizures. The lower number of BMs at diagnosis in hormone receptor-positive patients could be a possible explanation for the significant lower rate of headache and nausea in luminal Aand B-like patients. Further research must be performed to confirm these observations.

The strength of our analysis is the large sample size. To our knowledge, we analyzed the largest cohort of patients with asymptomatic BM of breast cancer. Moreover, we analyzed a homogeneous cohort of patients with BM of breast cancer and an absence of other malignant diseases. The limitation of the analysis is that a lead time bias of the earlier diagnosis of BM cannot be ruled out. A further limitation concerning the power of the multivariate analysis must be considered: Because of the missing data of performance status, multivariate logistic regression analysis could only be performed for 705 patients. The reason for a significant number of missing data is the retrospective and multicenter design of our subanalysis. An important characteristic that may have influenced the overall survival rates and performance status of patients is the existence of leptomeningeal metastases. Missing values 
for this parameter could be detected in 22 patients with asymptomatic BM and 23 patients with symptomatic BM.

Taking into account the retrospective design of this subanalysis, we cannot recommend a change in clinical practice concerning the management of patients with breast cancer without neurological complaints. The BM screening approach cannot be justified on the basis of our results. International guidelines have recommended no BM screening in breast cancer patients without neurological complaints, but a broad indication for brain imaging should be made if a patient has neurological symptoms related to BM. Our analysis is of clinical relevance in the context of prospective trials examining the benefit of early detection in breast cancer patients with a high risk for BM development. Considering the low time-dependent incidence of BM in early breast cancer patients [26,27], we suggest a prospective trial evaluating BM screening in patients with metastatic disease. It is estimated that almost a quarter of such patients develop BM [28]. A literature review performed by Komorowski et al. shows that in particular patients with HER2-positive and triple-negative metastatic breast cancer have a high incidence of BM (ranging between $22 \%$ and $36 \%$ and $15 \%$ and $37 \%$, respectively). Further risk factors for the development of BM among (HER positive) patients with metastatic breast cancer are younger age, progress of the metastatic breast cancer, and hormone receptor-negative disease [29]. There is less information available concerning risk factors for developing BM in patients with metastatic TNBC. Morris et al. [30] described a patient cohort with TNBC and BM in a follow-up time period: At the time of $\mathrm{BM}$ diagnosis, $68 \%$ of the patients had evidence of progression of extracranial disease. The analysis of Dawood et al. [31] showed that even patients with advanced TNBC without evidence of distant metastases (stage III) had a significantly higher risk of developing BM (hazards ratio vs. stage I 3.51; 95\% CI 1.85-6.67, $p=0.0001$ ). When designing a study evaluating BM early detection strategies, tumor subtype and breast cancer stage might help to identify patients at higher risk for developing BM. Biomarker evaluation for the prediction of BM should be integrated in ongoing studies with the perspective to select patients that are candidates for BM early detection.

\section{Materials and Methods}

The clinical data for the analysis was derived from the BMBC Registry. Patients with BM of breast cancer registered until April 2019 were considered for this analysis. The BMBC registry was approved by the ethic committee Hessen, Frankfurt am Main (ethic approval number FF42/2013).

The aim of the analysis was to characterize the patient cohort with BM of breast cancer with vs. without documented neurological symptoms. The following neurological symptoms of BM were assessed in the BMBC registry: seizure, nausea/vomiting, motor deficit, headache, visual disturbance, and mental health/psychological disturbance. The following characteristics for the cohort description were assessed: age at first diagnosis of breast cancer and of BM, number and localization of BM, biological subtype, ECOG/Karnofsky performance status at time of BM diagnosis, existence of leptomeningeal metastasis, maximum diameter of $\mathrm{BM}$ at first $\mathrm{BM}$ diagnosis, extracranial metastases $(\mathrm{ECM})$ at time of BM diagnosis, brain metastases-free interval (time from the first diagnosis of $\mathrm{BC}$ to the diagnosis of $\mathrm{BM}$ ), extracranial metastases-free survival (time from the first diagnosis of $\mathrm{BC}$ to ECM in the subgroup of patients with a diagnosis of BM after diagnosis of ECM), and local or contralateral recurrence-free interval (time from the first diagnosis of $\mathrm{BC}$ to local or contralateral recurrence) after diagnosis of breast cancer. Furthermore, we performed a comparison of patients with vs. without neurological symptoms regarding the local treatment of BM, year of diagnosis, diagnostic method of BM, and systemic treatment of breast cancer after the diagnosis of BM. Continuous data were summarized using the number of available data, mean, standard deviation (SD), median, and the minimum and maximum for each group. Categorical and ordinal data were summarized using the number and percentage of patients in each group. Differences in the categorical data between patients with symptomatic and asymptomatic BM data were tested by Chi-square or Fisher's exact test. Differences in the continuous variables between the two patient groups were tested non-parametrically using the Wilcoxon-Mann-Whitney test. For the analysis of the time intervals between the first 
diagnosis of $\mathrm{BC}$ to local or contralateral recurrence, cumulative incidence functions were plotted with death as a competing risk. Differences in the time intervals between symptomatic and asymptomatic patients were tested by Gray's test.

A multivariate logistic regression was performed for symptomatic vs. asymptomatic BM. The odds ratios with the corresponding 95\% confidence intervals were reported.

A further objective was to characterize the cohort of patients with neurological symptoms (seizure, nausea/vomiting, motor deficit, headache, impaired vision, mental health/ psychological disturbance) according to the biological subtype of the initial breast cancer. Biological subtype was defined as HER2-positive, TNBC (ER-, PR-negative, and HER2-negative) and luminal A- and B-like (luminal Aand B-like subtypes were evaluated together (ER and/or PR+, HER2-) because of the missing values of tumor grading causing an issue in the strict distinction of luminal A-like and luminal B-like patients).

Furthermore, we performed an analysis of the OS for the patients with and without neurological symptoms of BM. OS was defined as the time interval from the first diagnosis of BM to death due to any reason. Kapla-Meier curves and the median OS time with the corresponding 95\% confidence interval were determined. Differences in the survival curves were tested by the log-rank test. Additionally, an OS analysis in the different breast cancer subtypes (HER2-positive, triple-negative breast cancer, luminal A- and B-like) was performed.

All reported p-values were two-sided, and the significance level was set to 0.05 . Confidence intervals symmetrically cover $95 \%$. Adjustment for multiple testing was not planned.

The data was analyzed using SAS ${ }^{\circledR}$ (Statistical Analysis Software) version 9.4 with SAS Enterprise Guide Version 7.1 on Microsoft Windows 10 Enterprise.

\section{Conclusions}

Our analyses indicate that asymptomatic patients show a trend for a less severe metastatic disease in the brain and for a better outcome (statistically significant for a cohort of HER2-positive patients) despite a less intense local BM therapy compared to symptomatic patients. Although a lead time bias of the earlier diagnosis cannot be ruled out, this analysis is of potential clinical relevance in the context of potential trials examining the benefit of early detection of BM.

Author Contributions: Conceptualization, E.L., I.W. and V.M. (Volkmar Müller); Methodology, E.L., I.W., V.N., J.R. and V.M. (Volkmar Müller); Resources, T.N., R.W., M.S., T.-W.P.-S., V.M. (Volker Möbus), C.M., A.P., K.L., T.H., K.R., M.T., P.A.F., C.D., T.F. and S.L.; Software, V.N. and J.R.; Supervision, I.W., V.N., S.L. and V.M. (Volkmar Müller); Validation, E.L., I.W. and V.M. (Volkmar Müller); Visualization, V.N. and J.R.; Writing-original draft, E.L.; Writing-review and editing, I.W., T.N., R.W., M.S., T.-W.P.-S., V.M. (Volker Möbus), C.M., A.P., K.L., T.H., K.R., M.T., P.A.F., C.D., T.F., V.N., J.R., S.L. and V.M. (Volkmar Müller). All authors have read and agreed to the published version of the manuscript.

Funding: This research received no external funding.

Conflicts of Interest: V.M. (Volkmar Müller) received speaker honoraria from Amgen, Astra Zeneca, Daiichi-Sankyo, Eisai, Pfizer, MSD, Novartis, Roche, Teva, Seattle Genetics and consultancy honoraria from Genomic Health, Hexal, Roche, Pierre Fabre, Amgen, ClinSol, Novartis, MSD, Daiichi-Sankyo, Eisai, Lilly, Tesaro, Seattle Genetics and Nektar. Institutional research support from Novartis, Roche, Seattle Genetics, Genentech. Travel grants: Roche, Pfizer, Daiichi Sankyo. M.T. is a member of advisory Board of Amgen, AstraZeneca, Celgene, Daiichi Sankyo, Eisai, Exact Sciences, Lilly, MSD, Novartis, onkowissen.de, Pfizer, Pierre-Fabre, Roche, he provides manuscript support for Amgen, Celgene, Roche, received travel reimbursement from Amgen, Art Tempi, AstraZeneca, Celgene, Connect Medica, Daiichi Sankyo, Eisai, Exact Sciences, Hexal, I-Med-Institute, Lilly, MCI, MSD, Novartis, Omniamed, Pfizer, Roche, congress support from Amgen, AstraZeneca, Celgene, Daiichi Sanyko, Hexal, Novartis, Pfizer, Roche. M.T. is involved in lecture by Amgen, Art Tempi, AstraZeneca, Celgene, Connect Medica, Eisai, Exact Sciences, Hexal, I-Med-Institute, Lilly, MCI, MSD, Novartis, onkowissen.de, Omniamed, Pfizer, Roche, Vifor. M.T. received trial funding by Exact Sciences. M.S. received honoraria for speaker or consultancy role from AMGEN, AstraZeneca, Eisai, Lilly, Myelo Therapeutics, Novartis, Pantarhei Bioscience, Pfizer, Roche and Seattle Genetics. He received research funding from AstraZeneca, BioNTech, Eisai, Genentech, German Breast Group, Myelo Therapeutics, Novartis, Palleos, Pantarhei Bioscience, Pierre-Fabre, and Roche. He received travel reimbursement from Pfizer and Roche. P.F. reports personal fees from Novartis, Roche, Pfizer, Daiichi-Sankyo, Astra Zeneca, Eisai, Merck Sharp \& Dohme, Lilly, Pierre Fabre, Seattle, grants from Biontech, Cepheid, Genetics, during the conduct of the study. V. Möbus received speaker honoraria from Amgen, AstraZeneca, Celgene, Roche, Teva and consultancy honoraria from Roche, Amgen, Tesaro and Myelo Therapuetics. C.D. has stock 
and other ownership interests by Sividon Diagnostics (now Myriad), recieved honoraria by Novartis and Roche, has consulting or advisory role by MSD Oncology and Daiichi Sankyom, has patents, royalties or other intellectual property by VMScope digital pathology software, has two patents (patent application: EP18209672-cancer immunotherapy, patent application EP20150702464 - therapy response), received travel, accommodations, expenses from Roche, his institution received research funding by Myriad Genetics. S.L. has no conflict of interests concerning the presented analysis. S.L. has following relevant financial activities outside the submitted work (with conflict of interests): grants and honorario for lectures and ad boards paid to institute from Abbvie, Amgen, Celgene, Novartis, Pfizer, Roche; honorario for lectures and ad boards paid to institute from Seattle Genetics, Samsung, PriME/Medscape; personal fees for lecture from Chugai; grants from Teva, Vifor; grant and honorarium paid to institute from Daiichi-Sankyo; honorarium for ad boards paid to institute from Lilly; advisor honorarium paid to institute from Eirgenix, BMS, Puma; honorarium paid for institute from MSD, grant paid to institute from Immunomedics; grant and honorarium for lectures and ad boards paid to institute from Astra Zeneca. S.L. has intellectual property broadly relevant to the work (pending patent EP14153692.0 Immunsignature in TNBC). IW has no conflict of interest concerning the presented analysis, she received speaker's honoraria outside this work from Amgen, MSD, Novartis, Pierre Fabre Pharme, Pfizer, Roche, Sanofi-Aventis. Other authors declare no conflict of interest.

\section{References}

1. Meattini, I.; Andratschke, N. Challenges in the treatment of breast cancer brain metastases: Evidence, unresolved questions, and a practical algorithm. Clin. Transl. Oncol. 2020, 1-12. [CrossRef]

2. Witzel, I.; Oliveira-Ferrer, L. Breast cancer brain metastases: Biology and new clinical perspectives. Breast Cancer Res. 2016, 18, 1-9. [CrossRef]

3. Fisk, G.; Svensson, T. Incidence and time trends of brain metastases admissions among breast cancer patients in Sweden. Br. J. Cancer 2012, 106, 1850-1853. [CrossRef]

4. Darlix, A.; Louvel, G. Impact of breast cancer molecular subtypes on the incidence, kinetics and prognosis of central nervous system metastases in a large multicentre real-life cohort. Br. J. Cancer 2019, 121, 991-1000. [CrossRef] [PubMed]

5. Soni, A.; Ren, Z. Breast cancer subtypes predispose the site of distant metastases. Am. J. Clin. Pathol. 2015, 143, 471-478. [CrossRef] [PubMed]

6. Kennecke, H.; Yerushalmi, R. Metastatic behavior of breast cancer subtypes. J. Clin. Oncol. 2010, 28, 3271-3277. [CrossRef] [PubMed]

7. Sihto, H.; Lundin, J. Breast cancer biological subtypes and protein expression predict for the preferential distant metastasis sites: A nationwide cohort study. Breast Cancer Res. 2011, 13, R87. [CrossRef]

8. Smid, M.; Wang, Y. Subtypes of breast cancer show preferential site of relapse. Cancer Res. 2008, 68, 3108-3114. [CrossRef]

9. Lin, N.U.; Amiri-Kordestani, L. CNS metastases in breast cancer: Old challenge, new frontiers. Clin. Cancer Res. 2013, 19, 6404-6418. [CrossRef]

10. Witzel, I.; Laakmann, E. Treatment and outcomes of patients in the Brain Metastases in Breast Cancer Network Registry. Eur. J. Cancer 2018, 102, 1-9. [CrossRef]

11. Niikura, N.; Hayashi, N. Treatment outcomes and prognostic factors for patients with brain metastases from breast cancer of each subtype: A multicenter retrospective analysis. Breast Cancer Res. Treat. 2014, 147, 103-112. [PubMed]

12. Ramakrishna, N.; Temin, S. Recommendations on disease management for patients with advanced human epidermal growth factor receptor 2-Positive breast cancer and brain metastases: ASCO clinical practice guideline update. J. Clin. Oncol. 2018, 36, 2804-2807. [PubMed]

13. NCCN. Clinical Practice Guidelines in Oncology. Breast Cancer. Version 3.2020. Available online: https://www.nccn.org/store/login/login.aspx?ReturnURL=https://www.nccn.org/professionals/physician_ gls/pdf/breast.pdf (accessed on 25 September 2020).

14. Ditsch, N.; Untch, M. AGO recommendations for the diagnosis and treatment of patients with locally advanced and metastatic breast cancer: Update 2020. Breast Care 2020, 15, 294-309. [PubMed]

15. NCCN. Guidelines Small Cell Lung Cancer. Version 2.2020. Available online: https://www2.tri-kobe.org/ nccn/guideline/lung/english/small.pdf (accessed on 15 November 2019).

16. NCCN. Guidelines Non-Small Cell Lung Cancer. Version 3.2020. Available online: https://www2.tri-kobe. org/nccn/guideline/lung/english/non_small.pdf (accessed on 11 February 2020). 
17. Schouten, L.J.; Rutten, J. Incidence of brain metastases in a cohort of patients with carcinoma of the breast, colon, kidney, and lung and melanoma. Cancer 2002, 94, 2698-2705. [CrossRef]

18. Goncalves, P.H.; Peterson, S. Risk of brain metastases in patients with non-metastatic lung cancer: Analysis of the metropolitan detroit Surveillance, Epidemiology, and End Results (SEER) data. Cancer 2016, 122, 1921-1927. [CrossRef]

19. Barnholtz-Sloan, J.S.; Sloan, A.E. Incidence proportions of brain metastases in patients diagnosed (1973 to 2001) in the metropolitan Detroit cancer surveillance system. J. Clin. Oncol. 2004, 15, 2865-2872.

20. Chamberlain, M.C.; Baik, C.S. Systemic therapy of brain metastases: Non-small cell lung cancer, breast cancer, and melanoma. Neuro Oncol. 2017, 19, i1-i24.

21. Maurer, C.; Tulpin, L. Risk factors for the development of brain metastases in patients with HER2-positive breast cancer. ESMO Open 2018, 3, 1-9. [CrossRef]

22. Morikawa, A.; Wang, R. Characteristics and prognostic factors for patients with HER2-overexpressing breast cancer and brain metastases in the era of HER2-targeted therapy: An argument for earlier detection. Clin. Breast Cancer 2018, 18, 353-361. [CrossRef]

23. Lin, N.U.; Borges, V. Intracranial efficacy and survival with tucatinib plus trastuzumab and capecitabine for previously treated HER2-positive breast cancer with brain metastases in the HER2CLIMB trial. J. Clin. Oncol. 2020, 38, 1-10.

24. Wolpert, F.; Lareida, A. Risk factors for the development of epilepsy in patients with brain metastases. Neuro Oncol. 2019, 22, 718-728. [CrossRef] [PubMed]

25. Laakmann, E.; Witzel, I. Radiological patterns of brain metastases in breast cancer patients: A subproject of the German Brain Metastases in Breast Cancer (BMBC) registry. Int. J. Mol. Sci. 2016, 17, 1615. [CrossRef] [PubMed]

26. Laakmann, E.; Witzel, I. Development of central nervous system metastases as a first site of metastatic disease in breast cancer patients treated in the neoadjuvant trials GeparQuinto and GeparSixto. Breast Cancer Res. 2019, 21, 1-8. [CrossRef] [PubMed]

27. Komorowski, A.S.; Warner, E. Incidence of Brain metastases in nonmetastatic and metastatic breast cancer: Is there a role for screening? Clin. Breast Cancer 2020, 20, e54-e64. [CrossRef]

28. Pasquier, D.; Darlix, A. Treatment and outcomes in patients with central nervous system metastases from breast cancer in the real-life ESME MBC cohort. Eur. J. Cancer 2020, 125, 22-30. [CrossRef]

29. Hurvitz, S.A.; O'Shaughnessy, J. Central nervous system metastasis in patients with HER2-positive metastatic breast cancer: Patient characteristics, treatment, and survival from SystHERs. Clin. Cancer Res. 2019, 25, 2433-2441. [CrossRef]

30. Morris, P.G.; Murphy, C.G. Limited overall survival in patients with brain metastases from triple negative breast cancer. Breast J. 2012, 18, 345-350. [CrossRef] [PubMed]

31. Dawood, S.; Lei, X. Incidence of brain metastases as a first site of recurrence among women with triple receptor-negative breast cancer. Cancer 2012, 118, 4652-4659. [CrossRef]

(C) 2020 by the authors. Licensee MDPI, Basel, Switzerland. This article is an open access article distributed under the terms and conditions of the Creative Commons Attribution (CC BY) license (http://creativecommons.org/licenses/by/4.0/). 